\title{
Método híbrido de ejercicios de crossfit-halterofilia para el desarrollo de la fuerza-resistencia en Judo y Karate-do
}

Hybrid method of crossfit-halterophilia exercises for the development of force-resistance in Judo and karate-do

\section{Encịnas Trujillo, Manuel Octavio 1,2, Gavotto Nogales, Omar Iván ${ }^{1,2}$, Placencia Camacho, Lucia $^{2}$ y Antúnez Domínguez, Francisco Javier}

${ }^{1}$ Centro de Investigación y Educación Superior CIES-UNEPROP. Calle Boulevard José María Escriba 157, Villa del Palmar, C.P. 83105, Hermosillo, Sonora, México.

${ }^{2}$ Departamento de Ciencias del Deporte y de la Actividad Física. Universidad de Sonora. Calle Boulevard Luis Encinas s/n. Col centro, C.P. 83000, Hermosillo, Sonora, México.

\section{CORRESPONDENCIA}

Manuel Octavio Encinas Trujillo:

octavio.encinas@unison.mx

Centro de Investigación y Educación Superior

CIES-UNEPROP

\section{CÓMO CITAR}

Encinas, M., Gavotto, O., Placencia, L. \& Antúnez, F. (2020) Método híbrido de ejercicios de crossfit-halterofilia para el desarrollo de la fuerza-resistencia en Judo y Karate-do. Revista de Ciencias del Ejercicio, 15 (2), pp. 1-13. Recuperado de: revistafod.uanl.mx

\section{RESUMEN}

Se presenta una propuesta hibrida de entrenamiento con la aplicación de ejercicios de halterofilia y crossfit para el desarrollo de la fuerza - resistencia en los equipos de judo y karate do de la Universidad de Sonora. Se hace una primera validación, a través de un pre experimento de esta estrategia, alcanzándose resultados notables en los equipos de ambos deportes de la Universidad de Sonora. En la investigación se aplican un conjunto de métodos empíricos, a través del empleo de pruebas de fuerza para valorar el estado inicial de los atletas de alto rendimiento de judo y karate-do. Se registró un incrementó el desarrollo de fuerza y resistencia acompañado de la disminución de grasa corporal y aumentó la masa muscular en los deportistas, llegando a la conclusión que el programa desarrollado mediante la aplicación de acciones en estrategias hibridas es adecuada su aplicación en las etapas del entrenamiento deportivo de judo y karate do.

\section{ABSTRACT}

A hybrid training proposal is presented with the application of weightlifting and crossfit exercises for the development of strength - resistance in the judo and karate teams of the University of Sonora. A first validation is made, through a pre-experiment of this strategy, achieving remarkable results in the teams of both sports at the University of Sonora. In the research, a set of empirical methods are applied, through the use of strength tests to assess the initial state of high-performance judo and karate-do athletes. There was an increase in the development of strength and resistance accompanied by the decrease in body fat and increased muscle mass in athletes, reaching the conclusion that the program developed through the application of actions in hybrid strategies is suitable for application in the sports training of judo and karate-do.
Palabras clave: fuerza, resistencia, halterofilia, crossfit.
Key words: sstrength, endurance, weightlifting, crossfit. 
Así mismo se han constituido los modelos de enseñanza híbridos en los distintos programas educativos, pueden ser opciones muy interesantes en el campo deportivo, abordando los procesos de entrenamiento desde una dimensión bilateral en la que diversos modelos se complementen, creando una especie de simbiosis que permita a los atletas beneficiarse de las ventajas que cada modelo puede acarrear. Ante la demanda de innovación en los procesos de enseñanza-aprendizaje en el campo educativo, también en el área deportiva encontramos publicaciones con temas relacionados en la hibridación de los modelos de educación deportiva, responsabilidad personal y social; una experiencia a través de un programa de kickboxing educativo, corroborando que la combinación o hibridación de los modelos de educación deportiva, responsabilidad personal y social posibilita transformar los ambientes de aprendizaje en un contexto centrado en el estudiante. Así mismo, el kickboxing educativo (sin contacto) se ha mostrado como un contenido muy válido para la Educación Física (Menéndez y Fernández, 2016).

En estas participaciones se presentaron tres tipos de experiencia en construcción de este contexto hibrido, en el primer tipo la construcción de instalaciones lúdico-deportivas, a través de la colaboración y participación ciudadana de por medio, las cuales además de mostrarnos nuevos caminos creativos nos ilustran sobre interesantes oportunidades para una práctica deportiva que se inició en el ámbito del urbanismo participativo. En el segundo, apartado ejemplos de cómo el deporte se combina con las prácticas artísticas. Final mente, en el tercer apartado, otros tipos de proyectos referidos a deportivos híbridos con un carácter más tecnológico. Estos parten de la conexión entre el uso de las Tecnologías de la Información y Comunicación (TIC) con el deporte y las prácticas artísticas y creativas (Maza, 2015).

En este mismo contexto el plan estadounidense de alto rendimiento utiliza un método híbrido para los atletas que incorpora tres tipos principales de apoyo relacionados con el rendimiento. Estos tres métodos los proporcionan los Centros de Entrenamiento Olímpico de USOC en los Estados Unidos. Los atletas residentes están del $10 \%$ al $15 \%$ mejor que los otros atletas de su deporte (Moreau y Nabhan, 2012).

Es claro que el deporte de alto rendimiento está fundamentado en el alcance de logros, los cuales se convierten en el objetivo principal, tanto de los entrenadores como de los deportistas. Ello requiere de un proceso sistemático y multifactorial, el cual depende en gran medida de la relación existente, entre los procesos de entrenamiento, competencia y recuperación física (Issurin, 2012), así mismo podemos evidenciar que el deporte de alto rendimiento universitario es una actividad de entrenamiento y competición sistemática, altamente intensiva y científicamente fundamentada para alcanzar máximos resultados (Zhelyazkov, 2001).

Al respecto Matveet (2001) puntualiza, que los ejercicios a utilizar en la preparación del deportista deben modelar de una manera pronostica y real, los rasgos de la competición. En este entendimiento la preparación de los deportistas de judo y 
karate do demuestran que los ejercicios de crossfit y halterofilia son como los circuitos de resistencia y fuerza ya que se basan en el tiempo que dure su combate.

Es importante tener presente que judo y karate-do son deportes de combate de origen japonés que promueven un desarrollo integral, potenciando las capacidades físicas y psicomotoras, tales como la ubicación espacial, la perspectiva, el ambidextrismo, la lateralidad, la coordinación conjunta e independiente de ambas manos y pies, y la realización de acciones físicas diversas; así como de relación con otras personas, haciendo uso de técnicas en simulación de competición, con la lucha como un elemento integrador y dinamizador e introduciendo la iniciación técnico, táctico y deportiva de forma adaptada, además de buscar un acondicionamiento físico general e idóneo.

Coincidimos que para la aplicación de métodos híbridos en ambos deportes con respecto al componente físico; el desarrollo de la fuerza y resistencia, es necesario en estos deportes para el logro de metas; realizando las revisiones teóricas de este contexto, Iglesias (2000) puntualiza que la fuerza explosiva es la de mayor relevancia en el judo para desarrollarse, seguida por la fuerza máxima y la de resistencia, haciendo la sugerencia que la primera oscile entre un $30 \%$ a $60 \%$ en el entrenamiento, mientras que las otras dos un $30 \%$. En este mismo estudio, se identifica en judo los dos tipos de fuerza; la fuerza de resistencia, considerándola necesaria para las acciones defensivas, así como también la fuerza rápida o potencia para las acciones ofensivas; de tal manera al concluir el análisis de la utilización de estos tipos de fuerza, convenimos en la utilización en judo, la cual se puede determinar de acuerdo reconocimiento de los movimientos que demanda este deporte en la acción del combate; predominando el tiempo en relación al agarre, al ser el punto de partida para la aplicación de la fuerza rápida y la de resistencia; empleando la de resistencia con mayor énfasis en el meso ciclo de realización, para mejorar las manifestaciones de la fuerza al momento del combate (Hernández, 2018).

Por otra parte, en el karate-do se caracteriza por el empleo de diferentes técnicas de defensa y ataque, según la zona del cuerpo a defender o atacar. En el karate-do se unifican la fuerza, la rapidez, el control y el equilibrio, elementos que se resumen en el desarrollo de las capacidades físicas de fuerza y resistencia en el atleta a los fines de lograr resultados deportivos óptimos. Se busca derrotar al adversario mediante un impacto contundente, preciso y definitivo, buscando ser lo más eficaz posible. Este deporte también es considerado una manifestación dentro de uno mismo de la espontánea armonía con el universo, intentando cultivar la naturaleza humana ideal, la unión entre lo físico y lo espiritual a través del entrenamiento integral del cuerpo.

También se estipula el enfoque tradicional del karate do, enmarcado fundamentalmente en la utilización de la producción (nivel de asimilación) y la repetición como método, subutilizado en la parte inicial del aprendizaje como el de modelación, simulación y problematización, los cuales se complementan la contextualización de la enseñanza 
entendida como el proceso que motiva las relaciones del conocimiento con el contexto real del individuo y que lleva el conocimiento más allá, examinando las situaciones de otros contextos, analizando sus contradicciones y encuentros.

En ambos deportes predomina el perfil del deportista, con una perspectiva de introspección hacia el mismo, complementando la contextualización de la enseñanza y conocimiento hacia la realidad de los contextos en los encuentros; también prevalece en los dos, la búsqueda del logro en las competiciones para vencer al adversario; se puede cristalizar el logro del éxito, sumando las formas de enseñanza, técnicas y estrategias; así como también la técnica y táctica en acciones dominantes de la práctica, las cuales pueden contribuir a elevar las habilidades psicológicas al momento de la competición, consideradas desde la etapa inicial del entrenamiento, además que el deportista adquiera más seguridad en el mismo, añadiendo el perfeccionamiento integral; el resultado exitoso puede depender de cada uno de estos procesos en la planeación; solo sí, se respeta disciplinadamente los principios teóricos metodológicos científicos; además de la probabilidad de obtener mejores resultados al implementar estrategias hibridas de crossfit y halterofilia en el desarrollo de la fuerza y resistencia en ambos deportes.

El levantamiento de pesas es uno de los medios más utilizados dentro del entrenamiento de fuerza porque proporciona grandes incrementos de esta capacidad y por la facilidad de incorporar sus ejercicios a los entrenamientos (Núñez, 2018).
Sumado a esto, la implementación de ejercicios de crossfit, determinado como un sistema de entrenamiento de fuerza y acondicionamiento, basado en ejercicios funcionales constantemente variados y realizados a una alta intensidad. Por sus características se puede emplear en diversas disciplinas deportivas. Sus técnicas permiten combinarse de diversas maneras para intensificar el entrenamiento, elemento que le concede un valor excepcional para desarrollar las capacidades físicas de fuerza y resistencia.

Desde las últimas décadas del siglo pasado y con mayor énfasis desde los inicios del presente siglo, diversos estudios avalan la influencia del uso de la halterofilia y crossfit para desarrollar la fuerza y la resistencia en el entrenamiento deportivo, de manera específica en los deportes de combate (Sáenz, 2015; Chicaisa, 2017; Urrizaga, 2017).

Tanto en judo como en karate do, resulta trascendente el empleo de la fuerza-resistencia., los equipos de ambos deportes representativos de la Universidad de Sonora, trabajan con métodos y estrategias, que no incluyen el apoyo de acondicionamiento físico a través de ejercicios basados en halterofilia y de crossfit; es por eso la importancia de combinar esta batería de ejercicios para proveer numerosos beneficios a los entrenamientos para el logro de metas, entre los cuales se encuentra el aumento de la densidad ósea, los progresos en la coordinación muscular, el aprendizaje correcto de agarres y la mejora de la técnica de los ejercicios que se practican, así como el desarrollo de fuerza y resistencia; con la probabilidad de permitir a los deportistas de judo y de karate do de la Universidad de Sonora elevar su calidad deportiva, en la mejora de la técnicas para el logro de metas; descrito a continuación en el desarrollo metodológico. 
El objetivo fundamental del presente artículo es proponer un sistema de ejercicios o de actividades físicas de halterofilia y crossfit, para contribuir en la elevación de la fuerza y la resistencia en los deportes de judo y karate do de la Universidad de Sonora con indicaciones metodológicas de su aplicación que es lo que hemos llamado metodología de estrategia hibrida.

\section{Metodología}

Este estudio fundamenta en el enfoque cuantitativo, con base en un diseño pre-experimental.

Para comenzar la investigación se solicitó la colaboración a los dos entrenadores de judo y karate-do, para que permitieran que sus atletas participaran en el programa de fundamentos teórico- prácticos y metodológicos, para el proceso de desarrollo de la fuerza-resistencia, se les explico el propósito de este y los beneficios esperados, solicitando por escrito su consentimiento informado.

Accediendo a participar voluntariamente 12 atletas de alto rendimiento ocho de judo y cuatro de karate-do, todos de la categoría varonil en las diferentes modalidades, divisiones y pesos. La edad comprendida de los atletas se encuentra entre 18 y 25 años y que tienen menos de dos años entrenando y compitiendo.

Para el diagnóstico de la fuerza y resistencia en atletas de judo y karate-do, se aplicó una batería de evaluación con diferentes pruebas físicas que serían parte del diagnóstico de los atletas y que se repetirían para conocer al final del programa las modificaciones presentadas en el desarrollo de las capacidades físicas, específicamente fuerza y resistencia.

El método híbrido se estructuró por una combinación de ejercicios de crossfit y halterofilia que forma un sistema de ejercicios combinados de los citados anteriormente con indicaciones metodológicas para su aplicación en el entrenamiento deportivo de ambos deportes. de la estrategia hibrida propuesta. La estrategia hibrida se basó en las aportaciones de Conde y Delgado (2000) Glassman (2002) y Herrera (2017). De la investigación bibliográfica de los ejercicios de crossfit y halterofilia, también se observaron las secciones de entrenamiento de ambos deportes, más la opinión de los propios deportistas y entrenadores se seleccionaron seis ejercicios de halterofilia y ocho ejercicios de crossfit, siendo un total de 14 ejercicios para integrar la estrategia hibrida.

Los ejercicios se aplicaron en la etapa general del entrenamiento deportivo con repeticiones en series de tres, ocho, diez, por un periodo de tres meses, con una frecuencia de tres veces por semana durante 90 minutos para determinar el sistema de medición de la fuerza y la resistencia a los 12 deportistas.

A continuación, se presentan los ejercicios seleccionados:

Ejercicios de Fuerza en Halterofilia 1.- Thruster. El Thruster es un movimiento compuesto que combina estos dos ejercicios: sentadilla con press. 
2. - Puh Press con empuje de la barra. Con este ejercicio se trabaja glúteos, músculos isquiotibiales, músculos abdominales, hombros y tríceps. Al aplicar este ejercicio con alta carga a menos repeticiones para ganar fuerza de la cabeza a los pies o aligerar la carga y hacer más repeticiones a intervalos de tiempo para mejorar la fuerza-resistencia. Este ejercicio es para fortalecer las piernas y los brazos, por lo tanto, la combinación de ambos permite que el atleta obtenga mayor fuerza y resistencia.

3.- Back Squat (Sentadilla por Atrás) Este es uno de los ejercicios más importantes para desarrollar la fuerza muscular de las piernas. La sentadilla puede ser realizada a ritmo lento o rápido y también con detención en diferentes posiciones. En este ejercicio se trabaja los cuádriceps, glúteos y bíceps femoral.

4.- Deadlift (Peso Muerto) El levantamiento de peso muerto es uno de los ejercicios fundamentales de cadera. Con este ejercicio se fortalece la parte baja de la espalda, bícepfemorales y glúteos, con duración de segundos con una intensidad de grandes pesos para desarrollar la fuerza.

Ejercicios de Fuerza en Crossfitt 1.- Back Extensión (Lumbares) Con este ejercicio se trabaja espalda baja ya que es muy importante fortalecer la musculatura lumbar para el desarrollo de la fuerza. Para evitar lesiones las lumbares se realizan en colchonetas, se coloca boca abajo y partiendo de esta postura y concentrándonos en la zona lumbar se levanta a la vez la pierna derecha y el brazo opuesto, es decir, el izquierdo y luego se alterna la pierna izquierda y el brazo derecho y así sucesivamente el impacto en el resto de la espalda será mínimo. Se debe realizar cuatro series de 15 a 20 repeticiones por cada lado.

2.- Push Ups (Lagartijas) Es un ejercicio muy efectivo a alta intensidad desarrollando fuerza en los hombros, pecho, bíceps y tríceps. Este ejercicio consiste en levantar el cuerpo del suelo y ocupa varios grupos de músculos: Los pectorales y los tríceps son los que se han trabajado más a fondo.

\section{3.- Pull Ups (Dominadas) Con} Pull ups se demanda un gran esfuerzo a los músculos situados en la espalda alta y los brazos, pero al mismo tiempo, se ejercita la fuerza y se involucran otros músculos que contribuyen a mantener la postura del cuerpo recto. Los principales músculos trabajados son el dorsal ancho, redondo mayor, romboide, bíceps y braquial anterior.

Ejercicios de Resistencia en Halterofilia

1.- Snatch (Arranque) Arranque con repeticiones seguidas. Es un movimiento explosivo y de rápida ejecución, que consiste en llevar la barra desde el suelo hasta arriba de la 
cabeza, este ejercicio es de intensidad alta con descansos de 6 a 12 segundos. Este ejercicio Involucra diferentes grupos musculares y resulta un movimiento explosivo que permite ganar fuerza y potencia. Los principales músculos involucrados son los glúteos, isquiotibiales, cuádriceps, hombros, flexores de la cadera, lumbares y gemelos.

\section{2.- Clean and Jerk (Envión o 2 tiempos)} Los dos tiempos, cargada o envión, es en la halterofilia uno de los movimientos que forman parte de este deporte junto con el movimiento de arrancada. Los dos tiempos consiste en levantar la barra desde el suelo hasta los hombros con una sentadilla y repeticiones seguidas, con este ejercicio se trabaja sobre todo los flexores de la cadera, isquiotibiales, cuádriceps, deltoides, trapecio y en menor medida, gemelos y lumbares.

\section{Ejercicios de Resistencia en Crossfitt}

\section{1.-Montain Cleambers (Escaladores). -} Este es un movimiento de coordinación que fortalece el abdomen que consiste en llevar las rodillas a los codos. Con una intensidad moderada con descanso de 5 a 10 segundos. El ejercicio del escalador, escaladores, o mountain climbers, es un ejercicio completo que nos permite trabajar a nivel cardiovascular, con este ejercicio se trabaja piernas, abdomen, hombros y pecho y desarrollamos resistencia.

\section{2.- Jump Rope (Salto de Cuerda). Este} ejercicio es un trabajo cardio vascular y coordinación de piernas y brazos. El salto de cuerda es un buen método para mejorar la resistencia general e implica a muchas partes del cuerpo, sobre todo a los músculos del tren inferior del cuerpo que estarán activos todo el tiempo que dura el ejercicio. Esta actividad lo que hará es poner a prueba la resistencia de nuestro cuerpo, pues al implicar tantos músculos, el consumo de energía será elevado y el nivel de aguante del cuerpo será alto.

3.- Run (Correr) Distancia con 50 metro, máxima potencia, tiempo mínimo 12 segundos. Con este ejercicio se trabaja las piernas y se fortalece la resistencia. Los isquiotibiales, en cambio, tienen como función impulsarte hacia adelante gracias a la energía acumulada en el tendón de Aquiles.

4.- Wall Ball Shot (Lanzamiento de Balón). En este ejercicio se trabaja el tren inferior con una pelota medicinal con una intensidad alta y con descansos de cinco a 10 segundos. Con este ejercicio se trabajan músculos que participan en la acción de empuje, como hombros, pectorales y tríceps y los músculos del tren inferior involucrados en la sentadilla: glúteos, cuádriceps, isquiotibiales y en menor medida, aductores y abductores y músculos de la zona media del cuerpo.

5.- Ring Dips (Aros). Con este ejercicio se trabaja el equilibrio y fortalecimiento del tríceps, espalda, abdomen por la contracción. Entre los principales músculos trabajados se encuentran pectorales, tríceps, deltoides, trapecio y en menor medida, flexores de la muñeca y el recto abdominal que contribuye con la correcta postura y la estabilidad durante el movimiento. 
Resultados y discusión

Al realizar el análisis del comportamiento de los datos en los deportistas judocas participantes, los hallazgos más relevantes fue que de los ocho participantes con el programa hibrido, cinco incrementaron su capacidad de fuerza-resistencia y dos se mantuvieron igual sin cambios y uno disminuyo su capacidad, por no tener constancia en las sesiones de entrenamiento.

En los atletas de karate-do se apreció la elevación de la puntuación respecto a la fuerza y resistencia para la demanda de este deporte. En el análisis de estos cuatro deportistas del equipo de karate do; resultaron ser quienes obtuvieron los resultados más elevados en fuerza a diferencia de los judocas, así mismo podemos verificar que en este equipo en las diferentes variables trabajadas y evaluadas se obtuvieron incrementos en los resultados de los participantes.

Resultados de deportistas de karate-do:

JPR, inició con un peso corporal de 74.7 $\mathrm{kg}$, con un porcentaje de grasa de 20.6 $\%$ y muscular de $29.5 \%$ como primera medición antes de empezar el programa. Como primer test de fuerza en pecho en banca resultó una cantidad de $70 \mathrm{~kg}$ y en sentadilla $95 \mathrm{~kg}$. Con el seguimiento y las orientaciones técnicas superó esa marca en la segunda prueba $(75 \mathrm{~kg})$ y $95 \mathrm{~kg}$ en el tercer test de fuerza- resistencia. Alcanzó una marca de $100 \mathrm{~kg}$ de sentadilla y $80 \mathrm{~kg}$ pecho en banca respectivamente, igualando la fuerza del segundo ejercicio, mejorando su fuerza explosiva y resistencia a los combates cortos y su recuperación, gracias a las pruebas de 1600 metros, en la cual tuvo un resultado de 8 min con 23 segundo primer test, en el segundo 8 min con 5 segundos y en el tercero de 8 min con 32 segundos, dado este caso por el periodo de vacaciones que interrumpió el trascurso de este programa.

$\mathrm{FAH}$, fue uno de los atletas de una categoría de peso corporal mayor, con $104.5 \mathrm{~kg}$ de peso, porcentaje alto de grasa de $34.4 \%$ y muscular de $36.6 \%$. Tras el primer test de fuerza que registró un peso de $55 \mathrm{~kg}$ resultando menor a su peso corporal, se dio la tarea de estimular el aspecto de la alimentación y la exigencia durante el programa. Como resultante de ello se apreció un incremento no tanto en sus niveles de fuerza si no en cambio físico y de resistencia durante los combates, con pruebas físicas de resistencia de 1600 metros con tiempos de 10 min con 22 segundo, en la segunda prueba obtuvo 10 min con 11 segundos y en la tercera prueba 10 min con 20 segundos.

RMC, joven prospecto de la universidad de Sonora para futura medallas, registró un peso inicial de $77.5 \mathrm{~kg}$, una masa muscular de $36.5 \%$ y un porcentaje de grasa de $27.4 \%$, con unas pruebas iniciales de fuerza en pecho en banca de $65 \mathrm{~kg}$ y $85 \mathrm{~kg}$, en su segunda prueba de $70 \mathrm{~kg}$ y $95 \mathrm{~kg}$ y el tercer test de fuerza resistencia fue de $75 \mathrm{~kg}$ pecho en banca y $90 \mathrm{~kg}$ sentadilla, registrándose un aumento de 10 kilogramos totales en todo el programa, junto con la preparación de resistencia de 1600 metros de $10 \mathrm{~min}$ con 10 segundos, segunda prueba de 10 min con 22 segundos y en la tercera prueba de 10 min con 15 segundos.

$J C G$, con un peso corporal de $76.3 \mathrm{~kg}$, un porciento de grasa de $25.6 \%$ y un $32.4 \%$ de músculo. Se priorizó la tarea de bajar dichos niveles de grasa y aumentar los niveles de músculo. 
Mientras fueron avanzando las sesiones el atleta tuvo una mejoría de un total de peso de $74.7 \mathrm{~kg}$, bajando el porciento de grasa a $24.5 \%$ y subiendo el músculo a $34 \%$. También, al ejecutar las pruebas de fuerza y resistencia durante el programa; en los test de fuerza sus resultados fueron en aumento, para 12 kilogramos totales en las dos pruebas juntas y en resistencia su primera prueba fue de $7 \mathrm{~min}$ con $43 \mathrm{se}$ gundos, en la segunda logró una marca de 7 min con 36 segundos y en la tercera 7 min con 56 segundos.

En el análisis de estos cuatro deportistas del equipo de karate do; JPR y JCG, fueron quienes obtuvieron los resultados más elevados en fuerza a diferencia de FAH, RMC; con una diferencia de 15 puntos al final, así mismo podemos verificar que en este equipo en las diferentes variables trabajadas y evaluadas, se obtuvieron incrementos en los resultados en el $100 \%$ de los participantes.

Resultados de deportistas de judo

FLR, en su primera valoración y medición en la báscula (Tanita) arrojó un peso corporal de $99.400 \mathrm{~kg}$, razón por la cual la tarea fue enfocarse en bajar el porcentaje de grasa (27.7\%) y elevar el de músculo (40.2\%). El primer test de fuerza fue de $100 \mathrm{~kg}$ en pecho en banca y $95 \mathrm{~kg}$ en sentadilla. La segunda prueba fue de $110 \mathrm{~kg}$ y $100 \mathrm{~kg}$ respectivamente, mejorando en el tercer test de fuerza - resistencia con un resultado de $115 \mathrm{~kg}$ de pecho en banca y $105 \mathrm{~kg}$ en sentadilla. Este atleta no cumplió el $100 \%$ del programa que se aplicó, razón por la cual quedó por debajo del nivel de los demás atletas evaluados. En el desarrollo de la re- sistencia en 1600 metros (primer test) obtuvo 10 min con 45 segundos, en el segundo test 10 min 15 segundos y en el tercero 10 min 34 segundos. Hubo un lapso en el que se pausó el entrenamiento por motivo de vacaciones.

VRA, empezó con un nivel muy por debajo del promedio de sus compañeros judocas, en cuanto a niveles de fuerza, con un peso corporal de $73.9 \mathrm{~kg}$, razón por la cual se buscó la ganancia de peso corporal en músculo y la pérdida de grasa. Se empezó con el primer test de pecho en banca con $55 \mathrm{~kg}$ y en sentadilla $70 \mathrm{~kg}$. En el segundo test $60 \mathrm{~kg}$ y $80 \mathrm{~kg}$ respectivamente y en el tercer test de fuerza - resistencia $65 \mathrm{~kg}$ de pecho en banca y $85 \mathrm{~kg}$ de sentadilla, superando los obtenido en la medición anterior. El alumno fue constante en el programa por lo cual su desarrollo fue incrementado conforme fueron avanzado las sesiones del entrenamiento, mostrando una gran motivación, autoestima elevada, lo cual contribuyó al logro de una mejoría en su rendimiento físico, sin dejar atrás las pruebas de resistencia de 1600 metros. Con un tiempo en su primer test de $10 \mathrm{~min}$ con 15 segundos, en el segundo de 8 min 42 segundos y en el tercero de 8 min con 36 segundos.

$M M L$, inició con un peso corporal de $69 \mathrm{~kg}$. El objetivo buscado fue aumentar el peso corporal en ganancia en músculo y bajar el porcentaje de grasa. Por lo tanto, en las pruebas iniciales las evaluaciones de pecho en banca fue $90 \mathrm{~kg}$ y en sentadilla de $95 \mathrm{~kg}$. En el segundo test levantó $85 \mathrm{~kg}$ de pecho en banca y $95 \mathrm{~kg}$ de sentadilla, y en el tercero superó esto con $90 \mathrm{~kg}$ en pecho y $100 \mathrm{~kg}$ en sentadilla. 
Dando como observación un desarrollo mayor de la parte superior que la inferior, por lo tanto, el trabajo se enfocó más en el tren inferior. El atleta mejoró su composición corporal, bajando el porcentaje de grasa y subiendo el tono muscular. Los resultados de las pruebas de resistencia de 1600 metros fueron de $9 \mathrm{~min}$ con 22 segundos, en el segundo test 9 min con 10 segundos y en el tercero obtuvo 9 min 32 segundos.

MSV, como judoca de primer año en la Universidad de Sonora, nunca había desarrollado un programa formal de pesas (halterofilia y Crossfit). Se inició con un peso corporal de $72.7 \mathrm{~kg}$, un porcentaje de grasa de $20.9 \%$ y un tono muscular de $32.3 \%$; por lo que se enfocó el trabajo en el aumento del musculo, con test iniciales de pecho en banca de $65 \mathrm{~kg}$ y sentadilla $80 \mathrm{~kg}$.

Después de varias sesiones de entrenamiento, aunque no muy constante, hubo un registro en su segunda evaluación de un aumento de 5 kilos totales. Por otra parte, el tercer test de fuerza - resistencia resultó de $70 \mathrm{~kg}$ en pecho en banca y sentadilla $90 \mathrm{~kg}$ mejorando bastante. La primera prueba de resistencia registró 8 min con 48 segundos, en la segunda 8 min con 30 seg, y en la tercera 8 min con $45 \mathrm{seg}$ Se atribuye el incremento mostrado en la tercera prueba a su ausencia a las últimas sesiones del programa.

MRF, uno de los atletas que en la primera evaluación de fuerza inició con un peso corporal bajo de $63 \mathrm{~kg}$, con porciento de grasa mínimo de $18 \%$ y un tomo muscular de $30.5 \%$. Logró levantar en pecho en banca la cantidad de $85 \mathrm{~kg}$ y en sentadilla $95 \mathrm{~kg}$, sin embargo, posteriormente no bajo ni subió, se mantu- vo en los mismos rangos por una lesión anterior de rodilla y muñeca que impedía su total desempeño en el programa. El mismo le sirvió como rehabilitación para el fortalecimiento de esa lesión. En las pruebas de resistencia tuvo un tiempo inicial de 7 min con 22 segundos, segunda prueba de 7 min con 52 segundos por dicha inflamación de la rodilla y en su tercera prueba realizó 7 min con 36 segundos, mejorando así su nivel de resistencia respecto al segundo test.

$C L B$, como atleta de nuevo ingreso en la universidad nunca trabajó en forma híbrida o con un seguimiento o plan de entrenamiento establecido. La primera evaluación en medidas antropométricas fue de $97.7 \mathrm{~kg}$ de peso, con un porciento de grasa de $27.3 \%$ y de músculo de $37.6 \%$, registrando un nivel elevado de grasa en el cuerpo para un atleta de alto rendimiento. Por otro lado, en el primer test de fuerza en pecho en banca obtuvo $70 \mathrm{~kg}$ y en sentadilla $85 \mathrm{~kg}$ y en el segundo test se presentó un aumento de $10 \mathrm{~kg}$ totales como avance durante el seguimiento del programa, en el tercer test de fuerza - resistencia resultó un peso de $75 \mathrm{~kg}$ en pecho en banca y sentadilla $95 \mathrm{~kg}$. Por otro lado, el seguimiento de resistencia de 1600 metros fue de 11 min con 30 seg, en la segunda prueba el tiempo fue de 10 min con 50 seg., dando una mejoría en su capacidad de resistencia y en la tercera prueba fue de 10 min con 32 segundos, superando las dos pruebas iniciales.

$\mathrm{CRE}$, uno de los atletas con mejor técnica en el judo, puesto que obtuvo el bronce en la Universiada Nacional 2018 en Toluca, Edo. De México, en la categoría 100 $\mathrm{kg}$. En su primer test de pecho en banca obtuvo $90 \mathrm{~kg}$ y $105 \mathrm{~kg}$ en sentadilla. 
En el segundo test $90 \mathrm{~kg}$ y $100 \mathrm{~kg}$ respectivamente, resultados igualados en el tercer test. El programa se basó principalmente en el trabajo de resistencia de 1600 metros con unas pruebas iniciales de 9 min con 17 segundos, segundo test de 9 min con 20 sin mucha diferencia del primer test y en la tercera prueba con un resultado de 9 min con 50 segundos, este último se vio afectado por un malestar estomacal.

MBG, con un peso corporal de $82.3 \mathrm{~kg}$, un porcentaje de grasa de $26.7 \%$ y muscular de $34.2 \%$. Las pruebas de fuerza dieron un resultado en pecho en banca de $60 \mathrm{~kg}$ y sentadilla $75 \mathrm{~kg}$. Como segunda prueba con éxito, después de varias semanas del programa de acondicionamiento físico hibrido, logró un aumento de $15 \mathrm{~kg}$ totales en sus resultados anteriores, en el tercer test de fuerza.

Al realizar el análisis del comportamiento de los datos en los deportistas judocas participantes, los hallazgos más relevantes y diferencias fue que se concluyó que de los ocho participantes con el programa hibrido, cinco que representa un $62.5 \%$ incrementaron sus capacidad de resistencia-fuerza, dos que representa un $25 \%$ se mantuvieron igual sin cambios y uno que representa un $12.5 \%$ disminuyo su capacidad, por no tener disciplina en las sesiones de entrenamiento.

Los aspectos analizados permiten considerar, que se registró un incremento de la resistencia y la fuerza en los dos grupos de deportista, así como la disminución de grasa corporal y un aumento de la masa muscular en los deportistas de ambos deportes de combate, al realizar la aplicación del programa de estrategia hibrida.

\section{Conclusiones}

Al realizar la aplicación de esta estrategia hibrida con ejercicios de crossfit y halte- rofilia en los equipos de karate do y judo del deporte representativo de la Universidad de Sonora, podemos concluir que se incrementó la fuerza-resistencia de acuerdo con el objetivo del estudio. Los incrementos fueron graduales en el desarrollo del estudio, lo que permite sugerir que la metodología propuesta, puede ser una alternativa más para el apoyo en acciones de las planeaciones metodológicas del deporte de combate. Así mismo reflexionamos, la detección de limitaciones en el desarrollo de la fuerza-resistencia en los atletas de judo; confirmado esto, antes de la aplicación del proyecto de investigación, el insuficiente empleo de ejercicios de crossfit y halterofilia; la cual se sugiere implementar en los planes de entrenamiento y su dosificación de ejercicios; en igualdad de importancia en las cargas técnicas, tácticas y psicológicas, inmersos en el desarrollo planificado de las etapas de desarrollo del plan de entrenamiento ( etapa general, especifica, precompetitiva y en menor porcentaje en el periodo de transito) recomendado para los deporte del objeto de estudio y otros deportes.

En segundo término, concluimos que, al identificarse incremento de la resistencia y fuerza en los dos grupos de deportistas, también se demostró la probabilidad de disminuir la grasa corporal y un aumento de la masa muscular en los deportistas; el programa desarrollado mediante la aplicación de acciones en estrategias hibridas, puede ser un móvil para continuar con este tipo de investigaciones ampliando las variables en los deportes de combate, como a deportes individuales.

Por último, invitamos a la comunidad inmersa en esta área a continuar apoyando a las instituciones universitarias; así como a otros organismos que fomenten el deporte, para brindarles alternativas de sistemas de entrenamiento basados en la objetividad de la ciencia. 


\section{Referencias}

Conde, J. y Delgado, M. (2000). Características del proceso de entrenamiento. En Cárdenas, D. (Coord.). El entrenamiento integrado de las habilidades visuales en la iniciación deportiva. España: Ediciones Aljibe.

Chicaisa, H. (2017). La Halterofilia en el desarrollo muscular en los deportistas de Levantamiento de Pesas de la Federación Deportiva de Tungurahua. Proyecto de investigación. Editorial: Universidad Técnica de Ambato. Facultad de Ciencias Humanas y de la Educación. http://repositorio.uta.edu. ec/bitstream/123456789/25482/1/Hector\%200mar\%20Chicaisa\%20Ru mipamba-1804453684.pdf

Glassman, G. (2002). Guía de entrenamiento del nivel I de crossfit. Estados Unidos.

Hernández, R. (2018). Judo nuevas perspectivas sobre metodología y entrenamiento. España: Wanceulen. Recuperado de: https://books.google.com.mx/books?i$d=$ pe 5 iDwAAQBAJ\&printse $c=$ frontco-

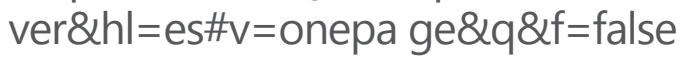

Herrera, J. (2017). Cross Training para usuarios avanzados. En: http://blog.fitness.decathlon.es/cross-training-para-usuarios-avanzados/

Issurin,V. B. (2012). Entrenamiento deportivo. Periodización en bloques. Barcelona: Paidotribo.

Iglesias, F. C. (2000). Propuesta de organización y control del entrenamiento de fuerza del judoka. acta de primer congreso. España.
Costorlenas, J. y Solé, J. (1997). El entrenamiento de la resistencia en los deportes de lucha con agarre: una propuesta integradora. Apunts Educación Física y Deportes, 81 -82. Obtenido de Revistes Catalanes amb Accés Obert (RACO): https://www.raco.cat/index.php/ApuntsEFD/article/view/311259

Maza, G. (2015). El deporte como un artefacto hibrido, bricolajes para la intervención social, cultural y creativa en el espacio público. Estudios Globales y Arte Contemporáneo, 86-88. https://revistes.ub.edu/ index.php/REGAC/article/view/14160

Matveet. (2001). Teoría General del Entrenamiento Deportivo. Barcelona: Paidotribo.

Menendez, J. y Fernández, J. (2016). Hibridación de los modelos de Educación Deportiva y Responsabilidad Personal y Social: una experiencia a través de un programa de kickboxing educativo. RETOS. Nuevas Tendencias en Educación Física, Deporte y Recreación. http://www.redalyc.org/articulo.oa?id $=345744747022$ ISSN 1579-1726

Moreau, J. y Nabhan, D. (2012). Organización y trabajo multidisciplinario en un centro olímpico de alto rendimiento en los estados unidos. Revista Médica Clínica Las Condes, 23(3). https://www. sciencedirect.com/science/article/pii/ S0716864012703206

Núñez, G. (2018). Levantamiento de pesas: Actualización técnica. Universidad de Sonora.

Sáenz, A. (2015). Creación de una fan page comunitaria en FB (Iglesias, 2000) y una guía impresa para la difusión de las actividades de los gimnasios de Crossfit. Proyecto de investigación. http://repositorio.ug.edu.ec/bitstream/redug/8814/1/ TESIS\%ANDRÉS\%20SÁENZ.pdf 
Salvatierra, G. y Izquierdo, E. (2014). Estudio del nuevo fenómeno deportivo Crossfit. http://buleria.unileon.es/bitstream/handle/10612/4185/8_SALVATIERRA_CAYETANO_GORKA_DICIEMBRE_2014.pdf? sequence $=1$

Urrizaga, M. (2017). Desarrollo de la fuerza en los deportes combate: Estudios relacionados en boxeo. Trabajo final integrador. Universidad Nacional de La Plata. Facultad de Humanidades y Ciencias de la Educación. En Memoria Académica. Disponible en: http:// www.memoria.fahce.unlp.edu.ar/tesis/ te.1319/te.1319.pdf

Zhelyazkov, T. (2001). Bases del entrenamiento deportivo. Barcelona. 